\title{
Analysis of star-disk interaction in young stellar systems
}

\author{
Nathalia N. J. Fonseca ${ }^{1,2}$, Silvia H. P. Alencar ${ }^{1}$ and Jérôme Bouvier ${ }^{2}$ \\ ${ }^{1}$ Departamento de Física - ICEx - UFMG, \\ Av. Antônio Carlos, 6627, 30270-901, Belo Horizonte, MG, Brazil \\ email: nath@fisica.ufmg.br \\ ${ }^{2}$ UJF-Grenoble 1/CNRS-INSU, Institut de Planétologie et d'Astrophysique de Grenoble \\ (IPAG), UMR 5274, Grenoble, F-38041, France
}

\begin{abstract}
We present preliminary results of the study of star-disk interaction in the classical T Tauri star V354 Mon, a member of the young stellar cluster NGC 2264. As part of an international campaign of observations of NGC 2264 organized from December 2011 to February 2012, high resolution photometric and spectroscopic data of this object were obtained simultaneously with the Chandra, CoRoT and Spitzer satellites, and ground-based telescopes, such as CFHT and ESO/VLT. The optical and infrared light curves of V354 Mon show periodic brightness minima that vary in depth and width every 5.21 days rotational cycle. We found evidence that the $\mathrm{H} \alpha$ emission line profile changes according to the period of photometric variations, indicating that the same phenomenon causes both modulations. Such correlation was also identified in a previous observational campaign on the same object, where we concluded that material non-uniformly distributed in the inner part of the disk is the main cause of the photometric modulation. This assumption is supported by the fact that the system is seen at high inclination. It is believed that this distortion of the inner part of the disk results from the dynamical interaction between the stellar magnetosphere, inclined with respect to the rotation axis, and the circumstellar disk, as also observed in the classical T Tauri star AA Tau, and predicted by magnetohydrodynamic numerical simulations. A model of occultation by circumstellar material was applied to the photometric data in order to determine the parameters of the obscuring material during both observational campaigns, thus providing an investigation of its stability on a timescale of a few years. We also studied V422 Mon, a classical T Tauri star with photometric variations similar to those of V354 Mon at optical wavelengths, but with a distinct behavior in the infrared. The mechanism that produces such a difference is investigated, testing the predictions of magnetospheric accretion models.
\end{abstract}

Keywords. stars: pre-main sequence, techniques: photometric, techniques: spectroscopic, accretion, accretion disks

\section{Introduction}

During the pre-main sequence phase, young $(\sim 1 \mathrm{Myr})$, low-mass $\left(\mathrm{M} \leqslant 2 \mathrm{M}_{\odot}\right)$ stars that exhibit signs of active accretion of material from its surrounding disk are classified as classical T Tauri stars (CTTSs). They present photometric and spectroscopic irregular variability, emission excess with respect to the stellar photosphere at wavelengths from $\mathrm{X}$-rays to the radio, broad emission lines with redshifted and blueshifted absorptions, and forbidden emission lines. These features are successfully explained by magnetospheric accretion models (Shu et al. 1994; Hartmann et al. 1994; Muzerolle et al. 2001; Kurosawa et al. 2006; Lima et al. 2010). In this scenario, the strong stellar magnetic field ( few kG) disrupts the disk at a distance of few stellar radii from the star. The inner disk material is then channeled at free-fall velocity along the dipole field lines, forming accretion funnels, where broad emission lines and redshifted absorption are generated. Hot spots are 
produced as material hits the photosphere, emitting an excess of optical and UV flux. An ionized disk wind is responsible for ejecting material from the system, producing blueshifted absorption and forbidden emission lines. The circumstellar disk reprocesses the radiation generated in the system, producing the observed infrared excess.

AA Tau is a well studied CTTS, observed for a month during three different campaigns (Bouvier et al. 1999, 2003, 2007). Its photometric modulation is characterized by an almost constant brightness level interrupted by quasi-cyclical and irregular episodes of attenuation. This peculiar behaviour is produced by a warp in the inner part of the circumstellar disk, which is caused by the interaction between the inner disk and the stellar magnetic field, tilted with respect to the rotation axis. The structure of the warp seems to evolve with time, as the photometric variation changes its shape on a timescale of a few weeks in each campaign to a few years from one campaign to the next. From an additional program of the CoRoT satellite that observed the young cluster NGC 2264 for 23 days uninterruptedly in March 2008, Alencar et al. (2010) identified that $28 \%$ of the observed CTTSs exhibited the same type of variability as AA Tau. This result indicates that occultation by circumstellar material as the main cause of photometric variability is common among young stars.

A few years ago, an international group of researchers organized a multiwavelength observational campaign of NGC 2264 with several satellites: CoRoT and MOST for 39 days in the optical, Spitzer for 29 days in the infrared, and Chandra for 3.5 days in the X-rays. The observations were simultaneously performed from December 2011 to January 2012. Data from ground-based telescopes were also obtained: spectroscopy with VLT/Flames for 20 nights, ur photometry with CFHT/Megacam for 15 nights in February 2012, I band with USNO for $~ 70$ nights from November 2011 to March 2012, and others. Covering simultaneously a wide range of wavelengths on a long timescale, this campaign will enable a comprehensive analysis of the physical processes that occur in young stars.

\section{V354 Mon}

V354 Mon, a K4V CTTS member of NGC 2264, was observed by CoRoT in both 2008 and 2011 campaigns and classified as AA Tau type. This star shows a periodic, but irregular photometric variability, as the depth and width of the minima change at each rotational cycle (Fig. 1, left). This morphological feature is present in the light
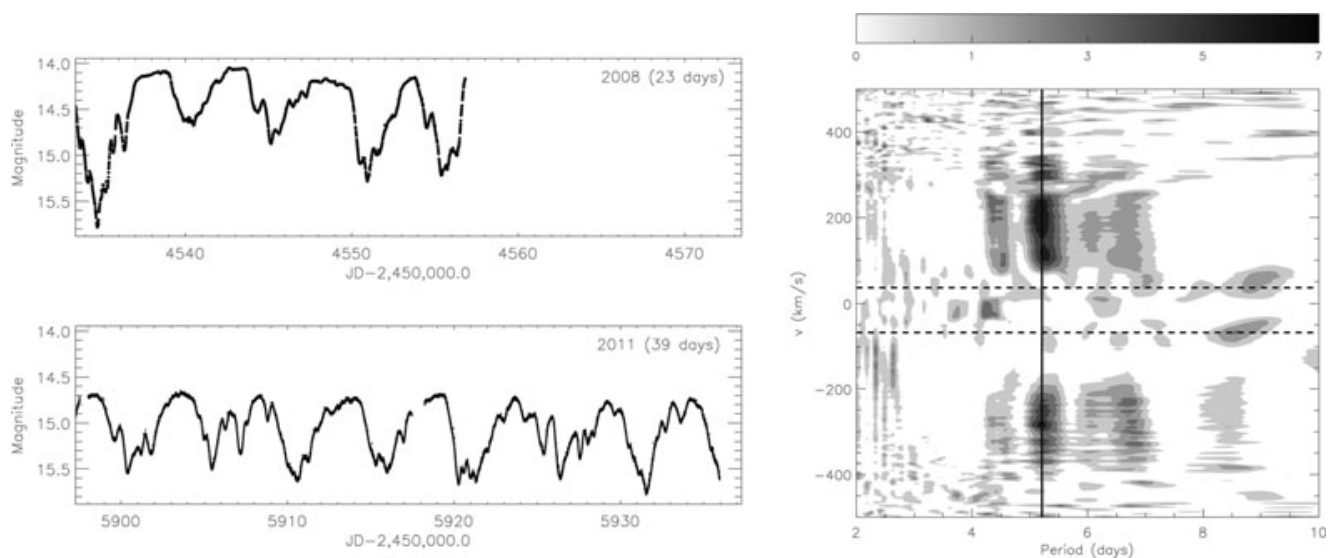

Figure 1. Left. CoRoT light curves obtained in 2008 (top) and 2011 (bottom). Right. Bidimensional periodogram of the $\mathrm{H} \alpha$ line flux. The horizontal dashed lines delimit the region dominated by nebular emission. The vertical solid line marks the period of 5.21 days. 

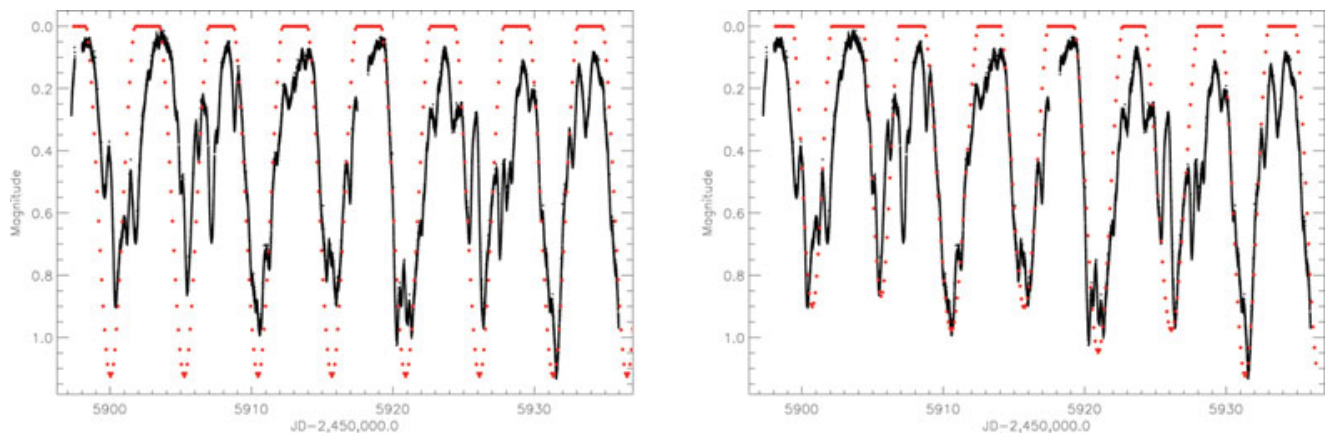

Figure 2. Best fit of the occultation model with fixed parameters (left) and individual fit of each CoRoT light curve minima (right) obtained in 2011.

curve of both campaigns. The photometric coverage for more than 100 days provided by combining CoRoT, CFHT, and USNO data led to a good determination of the period, $5.21 \pm 0.04$ days. This value is in agreement with the periods obtained from the 2008 CoRoT light curve, $5.26 \pm 0.50$ days, and by Lamm et al. (2004), $5.22 \pm 0.87$ days, indicating that the main cause of the photometric variability is stable over a few years.

During the 2008 CoRoT observations, simultaneous échelle spectroscopy was obtained for V354 Mon with the SOPHIE spectrograph at the Observatoire de Haute Provence (CNRS, France). We noticed that the emission lines vary in a cyclic manner according to the photometric modulation (Fonseca et al. in preparation). This correlation is also observed in the new campaign, as shown through the periodogram analysis of the $\mathrm{H} \alpha$ normalized flux (Fig. 1, right). Therefore, the same phenomenon produces the photometric and spectroscopic variability in V354 Mon.

Magnetohydrodynamic (MHD) simulations (Romanova et al. 2004) predict the formation of a warp in the inner disk when the magnetic axis is misaligned with respect to the rotation axis. As the stellar system rotates, the warp could occult periodically part of the stellar photosphere. This is a plausible scenario for V354 Mon, as this system is viewed at high inclination $\left(\sim 75^{\circ}\right)$ and shows the same type of variability as AA Tau. We applied a model of occultation by circumstellar material, originally developed for AA Tau (Bouvier et al. 1999), to the light curve of V354 Mon obtained in the 2011 campaign in order to determine the general parameters of the obscuring material. The warp, located at the corotation radius $\left(r_{c}\right)$, presented a maximum scale height $h / r_{c}$ of 0.33 and an azimuthal extension of $320^{\circ}$ (Fig. 2, left). Comparing with the parameters obtained in the model for the light curve of the 2008 campaign, 0.30 and $360^{\circ}$ (Fonseca et al. in preparation), we notice that the warp remained stable on a timescale of a few years. It is also interesting to observe that these characteristics are very similar to the ones obtained in the model of the variability of AA Tau (Bouvier et al. 1999).

The depth and width of V354 Mon minima vary at each rotational cycle, indicating that the warp changes its shape with time. This reveals a dynamical interaction between the stellar magnetosphere and the inner part of the disk (Goodson \& Winglee 1999). However, the parameters obtained from the individual fit of the model to the light curve minima are not very different from cycle to cycle (Fig. 2, right, and Table 1), indicating that the warp is a permanent structure.

\section{V422 Mon}

V422 Mon is a CTTS that presents light curve minima that vary in depth and width every rotational cycle, with a period of $8.93 \pm 0.92$ days. This similarity with V354 
Table 1. Occultation model parameters from individual fit of 2011 light curve minima

\begin{tabular}{l|ccccccc}
\multicolumn{7}{c}{$h(\phi)=h_{\max }\left|\cos \frac{\pi\left(\phi-\phi_{0}\right)}{2 \phi_{c}}\right|$} \\
\hline Minimum & $1^{\text {st }}$ & $2^{\text {nd }}$ & $3^{\text {rd }}$ & $4^{t h}$ & $5^{t h}$ & $6^{t h}$ & $7^{t h}$ \\
\hline$h_{\max }\left(r_{c}\right)$ & 0.31 & 0.30 & 0.31 & 0.31 & 0.32 & 0.31 & 0.33 \\
$2 \phi_{c}\left({ }^{\circ}\right)$ & 260 & 240 & 360 & 320 & 320 & 360 & 310 \\
\hline
\end{tabular}
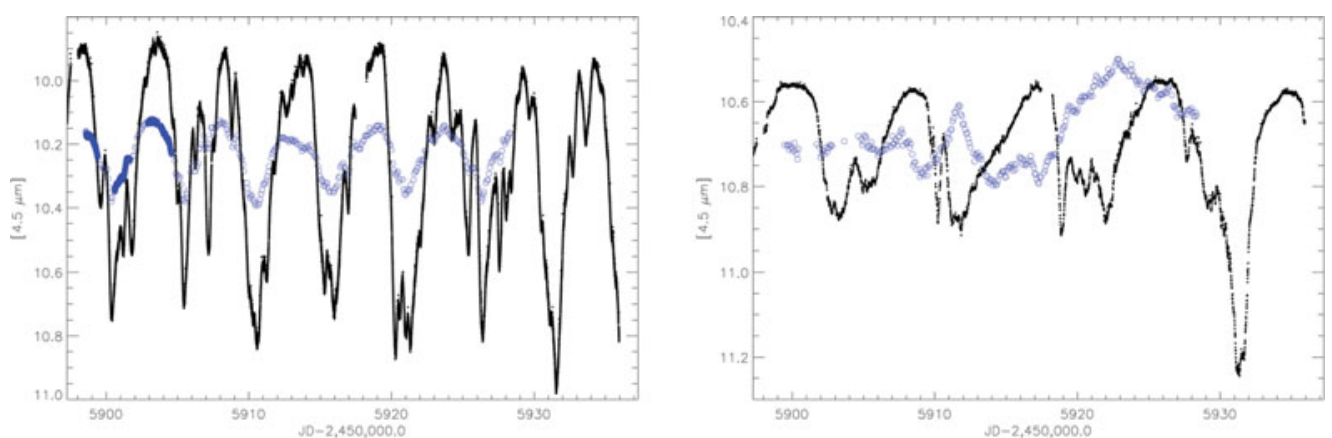

Figure 3. V354 Mon (left) and V422 Mon (right) CoRoT light curves (dots) obtained in 2011, compared with $4.5 \mu \mathrm{m}$ Spitzer data (open circle). CoRoT data was shifted to the mean of the Spitzer data.

Mon would indicate that both present a similar geometry. However, while the V354 Mon photometric modulation in the infrared is very similar to that seen in the optical, there is no agreement between the optical and infrared photometric variations of V422 Mon (Fig. 3). The optical modulation may be produced by a warp in the inner disk, as in V354 Mon, but the infrared variation may be caused by changes in the inner disk emission.

\section{Acknowledgement}

N. N. J. F. acknowledges support from CAPES (fellowship process $\mathrm{n}_{o}$. 18697-12-7), CNPq, CNES, and IAU. S. H. P. A. acknowledges support from CAPES, CNPq, and Fapemig. J. B. acknowledges support by ANR Toupies grant 2011 Blanc SIMI5-6 02001.

\section{References}

Alencar, S. H. P.., Teixeira, P. S., Guimarães, M. M., McGinnis, P. T., Gameiro, J. F., Bouvier, J., Aigrain, S., Flaccomio, E., \& Favata, F. 2010, A\&A A, 519, 88

Bouvier, J., Alencar, S. H. P.., Boutelier, T., Dougados, C., Balog, Z., Grankin, K., Hodgkin, S. T., Ibrahimov, M. A., Kun, M., Magakian, T. Y., \& Pinte, C. 2007, A\& A, 463, 1017

Bouvier, J., Chelli, A., Allain, S., Carrasco, L., Costero, R., Cruz-Gonzalez, I., Dougados, C., Fernández, M., Martín, E. L., Ménard, F., Mennessier, C., Mujica, R., Recillas, E., Salas, L., Schmidt, G., \& Wichmann, R. 1999, A\&A, 349, 619

Bouvier, J., Grankin, K. N., Alencar, S. H. P.., Dougados, C., Fernández, M., Basri, G., Batalha, C., Guenther, E., Ibrahimov, M. A., Magakian, T. Y., Melnikov, S. Y., Petrov, P. P., Rud, M. V., \& Zapatero Osorio, M. R. 2003, A\&\&A, 409, 169

Goodson, A. P. \& Winglee, R. M. 1999, ApJ, 524, 159

Hartmann, L., Hewett, R., \& Calvet, N. 1994, ApJ, 426, 669

Kurosawa, R., Harries, T. J., \& Symington, N. H. 2006, MNRAS, 370, 580

Lamm, M. H., Bailer-Jones, C. A. L.., Mundt, R., Herbst, W., \& Scholz, A. 2004, A\&A, 417, 557

Lima, G. H. R.. A., Alencar, S. H. P.., Calvet, N., Hartmann, L., \& Muzerolle, J. 2010, A\& A, 522, 104

Muzerolle, J., Calvet, N., \& Hartmann, L. 2001, ApJ, 550, 944

Romanova, M. M., Ustyugova, G. V., Koldoba, A. V., \& Lovelace, R. V. E.. 2004, ApJ, 610, 920

Shu, F., Najita, J., Ostriker, E., Wilkin, F., Ruden, S., \& Lizano, S. 1994, ApJ, 429, 781 